\title{
Some new inequalities of the Ostrowski-Grüss, Čebyšev, and Trapezoid types on time scales
}

\author{
Eze R. Nwaeze ${ }^{a, *}$, Nurhan Kaplan ${ }^{b}$, Fatma Gozde Tuna ${ }^{b}$, Adnan Tuna $^{b}$ \\ a Department of Mathematics, Tuskegee University, Tuskegee, AL 36088, USA. \\ ${ }^{b}$ Niğde Omer Halisdemir University, Art and Science Faculty, Mathematics Department, Niğde, Turkey.
}

\begin{abstract}
In this paper, we establish some novel Ostrowski-Grüss, Čebyšev, and Trapezoid type inequalities involving functions whose second derivatives are bounded on time scales. We also give some other interesting inequalities as special cases of our results.
\end{abstract}

Keywords: Ostrowski's inequality, Čebyšev inequality, Ostrowski-Grüss, Trapezoid inequality, time scales.

2010 MSC: 26D15, 54C30, 26D10.

(C)2019 All rights reserved.

\section{Introduction}

In 1938, Ostrowski [17] proved the following interesting integral inequality.

Theorem 1.1. Let $\mathrm{f}:[\mathrm{a}, \mathrm{b}] \rightarrow \mathbb{R}$ be continuous on $[\mathrm{a}, \mathrm{b}]$ and differentiable in $(\mathrm{a}, \mathrm{b})$ and its derivative $\mathrm{f}^{\prime}:(\mathrm{a}, \mathrm{b}) \rightarrow$ $\mathbb{R}$ be bounded in $(a, b)$. Then for any $x \in[a, b]$, we have

$$
\left|f(x)-\frac{1}{b-a} \int_{a}^{b} f(t) d t\right| \leqslant\left(\frac{1}{4}+\frac{\left(x-\frac{a+b}{2}\right)^{2}}{(b-a)^{2}}\right)(b-a)\left\|f^{\prime}\right\|_{\infty}
$$

where $\left\|f^{\prime}\right\|_{\infty}:=\sup _{x \in(a, b)}\left|f^{\prime}(x)\right|<\infty$. The inequality is sharp in the sense that the constant $\frac{1}{4}$ cannot be replaced by a smaller one.

In [3], Bohner and Matthews extended the above result to time scales by proving the succeeding theorem.

\footnotetext{
*Corresponding author

Email addresses: enwaeze@tuskegee.edu (Eze R. Nwaeze), nkaplan@ohu.edu.tr (Nurhan Kaplan), gtuna.com@gmail.com (Fatma Gozde Tuna), atuna@ohu.edu.tr (Adnan Tuna)

doi: $10.22436 /$ jnsa.012.04.01
}

Received: 2018-10-24 Revised: 2018-11-12 Accepted: 2018-11-15 
Theorem 1.2. Let $\mathrm{a}, \mathrm{b}, \mathrm{s}, \mathrm{t} \in \mathbb{T}, \mathrm{a}<\mathrm{b}$ and $\mathrm{f}:[\mathrm{a}, \mathrm{b}] \rightarrow \mathbb{R}$ be differentiable. Then for all $\mathrm{t} \in[\mathrm{a}, \mathrm{b}]$, we have

$$
\left|f(t)-\frac{1}{b-a} \int_{a}^{b} f(\sigma(s)) \Delta s\right| \leqslant \frac{M}{b-a}\left(h_{2}(t, a)+h_{2}(t, b)\right),
$$

where $h_{2}(.,$.$) is defined by Definition 2.11$ below and $M=\sup _{a<t<b}\left|f^{\Delta}(t)\right|<\infty$. This inequality is sharp in the sense that the right-hand side of (1.1) cannot be replaced by a smaller one.

If $f, g:[a, b] \rightarrow \mathbb{R}$ are integrable on $[a, b]$, then we consider the Čebyšev functional, $T(\cdot, \cdot)$, defined by

$$
T(f, g):=\frac{1}{b-a} \int_{a}^{b} f(t) g(t) d t-\left(\frac{1}{b-a} \int_{a}^{b} f(t) d t\right)\left(\frac{1}{b-a} \int_{a}^{b} g(t) d t\right) .
$$

In 1882, Čebyšev [7] proved that, if $f^{\prime}, g^{\prime} \in L_{\infty}[a, b]$, then

$$
|T(f, g)| \leqslant \frac{1}{12}(b-a)^{2}\left\|f^{\prime}\right\|_{\infty}\left\|g^{\prime}\right\|_{\infty} .
$$

In 1988, Hilger [9] introduced the time scales theory to unify continuous and discrete analysis. For some Ostrowski, Ostrowski-Grüss, Čebyšev, and Trapezoid type inequalities on time scales, see the papers [4, 8, 11-16, 19-21] where further references are provided.

In this present paper, we study some new Ostrowski-Grüss, Čebyšev, and Trapezoid type inequalities involving functions whose second derivatives are bounded on time scales. We also apply our results to the continuous and discrete cases.

\section{General definitions}

For a general introduction to the time scales theory we refer the reader to Hilger's Ph.D. thesis [9], the books $[5,6,10]$, and the survey [1].

Definition 2.1. A time scale $\mathbb{T}$ is an arbitrary nonempty closed subset of the real numbers.

We assume throughout that $\mathbb{T}$ has the topology that is inherited from the standard topology on $\mathbb{R}$. It is also assumed throughout that in $\mathbb{T}$ the interval $[a, b]$ means the set $\{t \in \mathbb{T}: a \leqslant t \leqslant b\}$ for the points $a<b$ in $\mathbb{T}$. Since a time scale may not be connected, we need the following concept of jump operators.

Definition 2.2. For each $t \in \mathbb{T}$, the forward jump operator $\sigma: \mathbb{T} \rightarrow \mathbb{T}$ is defined by $\sigma(t)=\inf \{s \in \mathbb{T}: s>t\}$ and the backward jump operator $\rho: \mathbb{T} \rightarrow \mathbb{T}$ is defined by $\rho(t)=\sup \{s \in \mathbb{T}: s<t\}$.

Definition 2.3. If $\sigma(t)>t$, then we say that $t$ is right-scattered, while if $\rho(t)<t$ then we say that $t$ is left-scattered. Points that are right-scattered and left-scattered at the same time are called isolated. If $\sigma(t)=t$, then $t$ is called right-dense, and if $\rho(t)=t$ then $t$ is called left-dense. Points that are both right-dense and left-dense are called dense.

Definition 2.4. The mapping $\mu: \mathbb{T} \rightarrow[0, \infty)$ defined by $\mu(t)=\sigma(t)-t$ is called the graininess function. The set $\mathbb{T}^{k}$ is defined as follows: if $\mathbb{T}$ has a left-scattered maximum $m$, then $\mathbb{T}^{k}=\mathbb{T}-\{m\}$; otherwise, $\mathbb{T}^{\mathrm{K}}=\mathbb{T}$.

If $\mathbb{T}=\mathbb{R}$, then $\mu(t)=0$, and when $\mathbb{T}=\mathbb{Z}$, we have $\mu(t)=1$. 
Definition 2.5. Let $f: \mathbb{T} \rightarrow \mathbb{R}$ and fix $t \in \mathbb{T}^{\kappa}$. Then we define $f^{\Delta}(t)$ to be the number (provided it exists) with the property that for any given $\epsilon>0$ there exists a neighborhood $U$ of $t$ such that

$$
\left|f(\sigma(t))-f(s)-f^{\Delta}(t)[\sigma(t)-s]\right| \leqslant \epsilon|\sigma(t)-s|, \quad \forall s \in U .
$$

We call $f^{\Delta}(t)$ the delta derivative of $f(t)$ at $t$. If the delta derivative of $f$ exists at each point in $\mathbb{T}^{k}$, we shall simply say that $f$ is differentiable.

In the case $\mathbb{T}=\mathbb{R}, f^{\Delta}(t)=\frac{d f(t)}{d t}$. In the case $\mathbb{T}=\mathbb{Z}, f^{\Delta}(t)=\Delta f(t)=f(t+1)-f(t)$, where $\Delta$ denotes the usual forward difference operator.

Theorem 2.6. If $\mathrm{f}, \mathrm{g}: \mathbb{T} \rightarrow \mathbb{R}$ are differentiable at $\mathrm{t} \in \mathbb{T}^{\mathrm{K}}$, then the product $\mathrm{fg}: \mathbb{T} \rightarrow \mathbb{R}$ is differentiable at $\mathrm{t}$ and

$$
(f g)^{\Delta}(t)=f^{\Delta}(t) g(t)+f(\sigma(t)) g^{\Delta}(t) .
$$

Definition 2.7. The function $f: \mathbb{T} \rightarrow \mathbb{R}$ is said to be rd-continuous on $\mathbb{T}$ provided it is continuous at all right-dense points $t \in \mathbb{T}$ and its left-sided limits exist at all left-dense points $t \in \mathbb{T}$. We denote the set of all rd-continuous functions from $\mathbb{T}$ into $\mathbb{R}$ by $C_{\text {rd }}(\mathbb{T}, \mathbb{R})$. Also, the set of functions $f: \mathbb{T} \rightarrow \mathbb{R}$ that are differentiable and whose second derivatives are rd-continuous is denoted by $C_{\text {rd }}^{2}(\mathbb{T}, \mathbb{R})$.

It follows from [4, Theorem 1.74] that every rd-continuous function has an antiderivative.

Definition 2.8. Let $F: \mathbb{T} \rightarrow \mathbb{R}$ be a function. Then $F: \mathbb{T} \rightarrow \mathbb{R}$ is called an antiderivative of $f$ on $\mathbb{T}$ if it satisfies $F^{\Delta}(t)=f(t)$ for any $t \in \mathbb{T}^{k}$. In this case the Cauchy integral is given by

$$
\int_{a}^{b} f(t) \Delta t=F(b)-F(a), \quad a, b \in \mathbb{T} .
$$

Theorem 2.9. Let $\mathrm{f}, \mathrm{g} \in \mathrm{C}_{\mathrm{rd}}(\mathbb{T}, \mathbb{R}), \mathrm{a}, \mathrm{b}, \mathrm{c} \in \mathbb{T}$ and $\alpha, \beta \in \mathbb{R}$. Then

1. $\int_{a}^{b}[\alpha f(t)+\beta g(t)] \Delta t=\alpha \int_{a}^{b} f(t) \Delta t+\beta \int_{a}^{b} g(t) \Delta t ;$

2. $\int_{a}^{b} f(t) \Delta t=-\int_{b}^{a} f(t) \Delta t$

3. $\int_{a}^{b} f(t) \Delta t=\int_{a}^{c} f(t) \Delta t+\int_{c}^{b} f(t) \Delta t$

4. $\int_{a}^{b} f(t) g^{\Delta}(t) \Delta t=(f g)(b)-(f g)(a)-\int_{a}^{b} f^{\Delta}(t) g(\sigma(t)) \Delta t$.

Theorem 2.10. If $\mathrm{f}$ is $\Delta$-integrable on $[\mathrm{a}, \mathrm{b}]$, then so is $|\mathrm{f}|$, and

$$
\left|\int_{a}^{b} f(t) \Delta t\right| \leqslant \int_{a}^{b}|f(t)| \Delta t
$$

Definition 2.11. Let $h_{k}, g_{k}: \mathbb{T}^{2} \rightarrow \mathbb{R}, k \in \mathbb{N}_{0}$ be defined by $h_{0}(t, s):=g_{0}(t, s):=1$ for all $s, t \in \mathbb{T}$ and then recursively by $g_{k+1}(t, s)=\int_{s}^{t} g_{k}(\sigma(\tau), s) \Delta \tau, h_{k+1}(t, s)=\int_{s}^{t} h_{k}(\tau, s) \Delta \tau$ for all $s, t \in \mathbb{T}$.

\section{Main results}

The following theorem deals with Ostrowski-Grüss type inequalities on time scales. 
Theorem 3.1. Let $\mathrm{a}, \mathrm{b}, \mathrm{t}, \mathrm{x} \in \mathbb{T}, \mathrm{a}<\mathrm{b}$, and $\mathrm{f} \in \mathrm{C}_{\mathrm{rd}}^{2}(\mathbb{T}, \mathbb{R})$. Then, for all $\mathrm{x} \in[\mathrm{a}, \mathrm{b}]$, we have following inequalities:

$$
\begin{aligned}
\mid f(x) & -\frac{1}{b-a} \int_{a}^{b} f(t) \Delta t-\left(x-b+\frac{g_{2}(b, a)}{b-a}\right) f^{\Delta}(x) \\
& +\left(\frac{g_{3}(b, a)}{b-a}+(x-b) \frac{g_{2}(b, a)}{b-a}+h_{2}(b, x)\right)\left(\frac{f^{\Delta}(b)-f^{\Delta}(a)}{b-a}\right) \mid \\
\leqslant & {\left[\frac{1}{b-a}\left(\int_{a}^{x} g_{2}^{2}(\sigma(t), a) \Delta t+\int_{x}^{b} h_{2}^{2}(b, \sigma(t)) \Delta t\right)-\left(\frac{g_{3}(b, a)}{b-a}+(x-b) \frac{g_{2}(b, a)}{b-a}+h_{2}(b, x)\right)^{2}\right]^{\frac{1}{2}} } \\
& \times\left[\frac{1}{b-a} \int_{a}^{b}\left(f^{\Delta \Delta}(t)\right)^{2} \Delta t-\left(\frac{f^{\Delta}(b)-f^{\Delta}(a)}{b-a}\right)^{2}\right]^{\frac{1}{2}},
\end{aligned}
$$

and

$$
\begin{aligned}
\mid f(x) & -\frac{2}{b-a} \int_{a}^{b} f(t) \Delta t+\frac{g_{2}(b, a) f(a)+h_{2}(b, a) f(b)}{(b-a)^{2}}-\left(x-b+\frac{g_{2}(b, a)}{b-a}\right) f^{\Delta}(x) \\
& +\left(2 g_{3}(x, a)-\frac{g_{2}(b, a)}{b-a} g_{2}(x, a)-2 g_{3}(x, b)-\frac{h_{2}(b, a)}{b-a} g_{2}(x, b)\right)\left(\frac{f^{\Delta}(b)-f^{\Delta}(a)}{(b-a)^{2}}\right) \mid \\
\leqslant & \frac{1}{b-a}\left[\frac { 1 } { b - a } \left(\int_{a}^{x}\left(2(b-a) g_{2}(\sigma(t), a)-(\sigma(t)-a) g_{2}(b, a)\right)^{2} \Delta t\right.\right. \\
& \left.+\int_{x}^{b}\left(2(b-a) h_{2}(b, \sigma(t))-(b-\sigma(t)) h_{2}(b, a)\right)^{2} \Delta t\right) \\
& \left.\left.-\left(2 g_{3}(x, a)-\frac{g_{2}(b, a)}{b-a} g_{2}(x, a)-2 g_{3}(x, b)-\frac{h_{2}(b, a)}{b-a} g_{2}(x, b)\right)^{2}\right]^{\frac{1}{2}}\right] \\
& \times\left[\frac{1}{b-a} \int_{a}^{b}\left(f^{\Delta \Delta}(t)\right)^{2} \Delta t-\left(\frac{f^{\Delta}(b)-f^{\Delta}(a)}{b-a}\right)^{2}\right]^{\frac{1}{2}} .
\end{aligned}
$$

Proof. We have

$$
\begin{aligned}
& \frac{1}{2(b-a)^{2}} \int_{a}^{b} \int_{a}^{b}(u(x, \sigma(t))-u(x, \sigma(s)))\left(f^{\Delta \Delta}(t)-f^{\Delta \Delta}(s)\right) \Delta t \Delta s \\
& =\frac{1}{b-a} \int_{a}^{b} u(x, \sigma(t)) f^{\Delta \Delta}(t) \Delta t-\left(\frac{1}{b-a} \int_{a}^{b} u(x, \sigma(t)) \Delta t\right)\left(\frac{1}{b-a} \int_{a}^{b} f^{\Delta \Delta}(t) \Delta t\right) .
\end{aligned}
$$

We have (see also [2, pp. 7])

$$
\frac{1}{b-a} \int_{a}^{b} u(x, \sigma(t)) f^{\Delta \Delta}(t) \Delta t=\frac{1}{b-a} \int_{a}^{b} f(t) \Delta t+\left(x-b+\frac{g_{2}(b, a)}{b-a}\right) f^{\Delta}(x)-f(x),
$$


where

$$
u(x, \sigma(t))= \begin{cases}g_{2}(t, a), & t \in[a, x), \\ h_{2}(b, t), & t \in[x, b] .\end{cases}
$$

Additionally, we get

$$
\frac{1}{b-a} \int_{a}^{b} f^{\Delta \Delta}(t) \Delta t=\frac{f^{\Delta}(b)-f^{\Delta}(a)}{b-a}
$$

and

$$
\frac{1}{b-a} \int_{a}^{b} u(x, \sigma(t)) \Delta t=\frac{g_{3}(b, a)}{b-a}+(x-b) \frac{g_{2}(b, a)}{b-a}+h_{2}(b, x) .
$$

Using the Cauchy-Schwartz inequality, we may write

$$
\begin{aligned}
& \left|\frac{1}{2(b-a)^{2}} \int_{a}^{b} \int_{a}^{b}(u(x, \sigma(t))-u(x, \sigma(s)))\left(f^{\Delta \Delta}(t)-f^{\Delta \Delta}(s)\right) \Delta t \Delta s\right| \\
& \leqslant\left(\frac{1}{2(b-a)^{2}} \int_{a}^{b} \int_{a}^{b}(u(x, \sigma(t))-u(x, \sigma(s)))^{2} \Delta t \Delta s\right)^{\frac{1}{2}}\left(\frac{1}{2(b-a)^{2}} \int_{a}^{b} \int_{a}^{b}\left(f^{\Delta \Delta}(t)-f^{\Delta \Delta}(s)\right)^{2} \Delta t \Delta s\right)^{\frac{1}{2}} .
\end{aligned}
$$

Therefore,

$$
\begin{aligned}
& \frac{1}{2(b-a)^{2}} \int_{a}^{b} \int_{a}^{b}(u(x, \sigma(t))-u(x, \sigma(s)))^{2} \Delta t \Delta s \\
& =\frac{1}{b-a} \int_{a}^{b} u^{2}(x, \sigma(t)) \Delta t-\left(\frac{1}{b-a} \int_{a}^{b} u(x, \sigma(t)) \Delta t\right)^{2} \\
& =\frac{1}{b-a}\left(\int_{a}^{x} g_{2}^{2}(\sigma(t), a) \Delta t+\int_{x}^{b} h_{2}^{2}(b, \sigma(t)) \Delta t\right)-\left(\frac{g_{3}(b, a)}{b-a}+(x-b) \frac{g_{2}(b, a)}{b-a}+h_{2}(b, x)\right)^{2},
\end{aligned}
$$

and

$$
\frac{1}{2(b-a)^{2}} \int_{a}^{b} \int_{a}^{b}\left(f^{\Delta \Delta}(t)-f^{\Delta \Delta}(s)\right)^{2} \Delta t \Delta s=\frac{1}{b-a} \int_{a}^{b}\left(f^{\Delta \Delta}(t)\right)^{2} \Delta t-\left(\frac{1}{b-a} \int_{a}^{b} f^{\Delta \Delta}(t) \Delta t\right)^{2} .
$$

Using (3.3)-(3.9), we can easily obtain the following inequality

$$
\begin{aligned}
& \mid f(x)-\frac{1}{b-a} \int_{a}^{b} f(t) \Delta t-\left(x-b+\frac{g_{2}(b, a)}{b-a}\right) f^{\Delta}(x) \\
& +\left(\frac{g_{3}(b, a)}{b-a}+(x-b) \frac{g_{2}(b, a)}{b-a}+h_{2}(b, x)\right)\left(\frac{f^{\Delta}(b)-f^{\Delta}(a)}{b-a}\right) \mid \\
& \leqslant\left[\frac{1}{b-a}\left(\int_{a}^{x} g_{2}^{2}(\sigma(t), a) \Delta t+\int_{x}^{b} h_{2}^{2}(b, \sigma(t)) \Delta t\right)-\left(\frac{g_{3}(b, a)}{b-a}+(x-b) \frac{g_{2}(b, a)}{b-a}+h_{2}(b, x)\right)^{2}\right]^{\frac{1}{2}} \\
& \quad \times\left[\frac{1}{b-a} \int_{a}^{b}\left(f^{\Delta \Delta}(s)\right)^{2} \Delta s-\left(\frac{f^{\Delta}(b)-f^{\Delta}(a)}{b-a}\right)^{2}\right]^{\frac{1}{2}},
\end{aligned}
$$


which provides the first inequality in (3.1).

We also have (see [2, pp.11])

$$
\begin{aligned}
\frac{1}{b-a} \int_{a}^{b} u(x, \sigma(t)) f^{\Delta \Delta}(t) \Delta t= & 2 \int_{a}^{b} f(t) \Delta t-\frac{g_{2}(b, a) f(a)+h_{2}(b, a) f(b)}{b-a} \\
& +(b-a)\left(x-b+\frac{g_{2}(b, a)}{b-a}\right) f^{\Delta}(x)-(b-a) f(x),
\end{aligned}
$$

where

$$
u(x, \sigma(t))= \begin{cases}2(b-a) g_{2}(t, a)-(t-a) g_{2}(b, a), & t \in[a, x), \\ 2(b-a) h_{2}(b, t)-(b-t) h_{2}(b, a), & t \in[x, b]\end{cases}
$$

Additionally, we can obtain

$$
\frac{1}{b-a} \int_{a}^{b} u(x, \sigma(t)) \Delta t=2 g_{3}(x, a)-\frac{g_{2}(b, a)}{b-a} g_{2}(x, a)-2 g_{3}(x, b)-\frac{h_{2}(b, a)}{b-a} g_{2}(x, b) .
$$

However

$$
\begin{aligned}
\frac{1}{2(b-a)^{2}} \int_{a}^{b} \int_{a}^{b}(u(x, \sigma(t))-u(x, \sigma(s)))^{2} \Delta t \Delta s \\
=\frac{1}{b-a} \int_{a}^{b} u^{2}(x, \sigma(t)) \Delta t-\left(\frac{1}{b-a} \int_{a}^{b} u(x, \sigma(t)) \Delta t\right)^{2} \\
=\frac{1}{b-a}\left[\int_{a}^{x}\left(2(b-a) g_{2}(\sigma(t), a)-(\sigma(t)-a) g_{2}(b, a)\right)^{2} \Delta t\right. \\
\left.+\int_{x}^{b}\left(2(b-a) h_{2}(b, \sigma(t))-(b-\sigma(t)) h_{2}(b, a)\right)^{2} \Delta t\right] \\
-\left(2 g_{3}(x, a)-\frac{g_{2}(b, a)}{b-a} g_{2}(x, a)-2 g_{3}(x, b)-\frac{h_{2}(b, a)}{b-a} g_{2}(x, b)\right)^{2} .
\end{aligned}
$$

Using (3.3), (3.5), (3.7), (3.9), (3.10), and (3.11), we can obtain the inequality (3.2). This completes the proof of the theorem.

Corollary 3.2. By taking $\mathbb{T}=\mathbb{R}$ in Theorem 3.1, we get

$$
\begin{aligned}
& \left|f(x)-\frac{1}{b-a} \int_{a}^{b} f(t) d t-\left(x-\frac{a+b}{2}\right) f^{\prime}(x)+\frac{1}{2}\left(\left(t-\frac{a+b}{2}\right)^{2}+\frac{(b-a)^{2}}{12}\right)\left(\frac{f^{\prime}(b)-f^{\prime}(a)}{b-a}\right)\right| \\
& \leqslant\left[\frac{1}{180}(b-a)^{2}\left(4(a+b)^{2}-15 x(a+b-x)-a b\right)\right]^{\frac{1}{2}}\left[\frac{1}{b-a} \int_{a}^{b}\left(f^{\prime \prime}(t)\right)^{2} d t-\left(\frac{f^{\prime}(b)-f^{\prime}(a)}{b-a}\right)^{2}\right]^{\frac{1}{2}},
\end{aligned}
$$

and

$$
f(x)-\frac{2}{b-a} \int_{a}^{b} f(t) d t+\frac{f(a)+f(b)}{2}-\left(x-\frac{a+b}{2}\right) f^{\prime}(x)
$$




$$
\begin{aligned}
& +\left(\frac{1}{12}(b-a)\left((a+b)^{2}-6 x(a+b-x)+2 a b\right)\right)\left(\frac{f^{\prime}(b)-f^{\prime}(a)}{(b-a)^{2}}\right) \mid \\
\leqslant & \frac{1}{b-a}\left[\frac { 1 } { 7 2 0 } ( b - a ) ^ { 2 } \left(180 x^{4}-360(a+b) x^{3}+300(a+b)^{2} x^{2}-120(a+b)^{3} x\right.\right. \\
& \left.\left.+19(a+b)^{4}-4 a b\left(8(a+b)^{2}+30\left(x^{2}-x(a+b)\right)-a b\right)\right)\right]^{\frac{1}{2}} \\
& \times\left[\frac{1}{(b-a)^{2}} \int_{a}^{b}\left(f^{\prime \prime}(t)\right)^{2} d t-\left(\frac{f^{\prime}(b)-f^{\prime}(a)}{b-a}\right)^{2}\right]^{\frac{1}{2}} .
\end{aligned}
$$

Corollary 3.3. For $\mathbb{T}=\mathbb{Z}$ in Theorem 3.1 , we have

$$
\begin{aligned}
& \mid f(x)-\frac{1}{b-a} \sum_{t=a}^{b-1} f(t)-\left(x-\frac{a+b-1}{2}\right) \Delta f(x) \\
& \quad+\frac{1}{2}\left(\left(x+1-\frac{a+b}{2}\right)^{2}+\frac{(b-a+2)(b-a-2)}{12}\right)\left(\frac{\Delta f(b)-\Delta f(a)}{b-a}\right) \mid \\
& \leqslant\left[\frac{1}{180}\left((b-a)^{2}-1\right)\left(15\left((x+1)^{2}-(x+1)(a+b)\right)+4(a+b)^{2}-a b-1\right)\right]^{\frac{1}{2}} \\
& \quad \times\left[\frac{1}{b-a} \sum_{t=a}^{b-1}\left(\Delta^{2} f(t)\right)^{2}-\left(\frac{\Delta f(b)-\Delta f(a)}{b-a}\right)^{2}\right]^{\frac{1}{2}},
\end{aligned}
$$

and

$$
\begin{aligned}
& \mid f(x)-\frac{2}{b-a} \sum_{t=a}^{b-1} f(t)+\frac{(b-a+1) f(a)+(b-a-1) f(b)}{2(b-a)}-\left(x-\frac{a+b-1}{2}\right) \Delta f(x) \\
& \quad+\left(\frac{1}{12}(b-a)\left((a+b)^{2}-6(a+b)(x+1)+6 x^{2}+2 a b+12 x+5\right)\right)\left(\frac{\Delta f(b)-\Delta f(a)}{(b-a)^{2}}\right) \mid \\
& \leqslant \\
& \quad \frac{1}{b-a}\left[\frac { 1 } { 7 2 0 } ( b - a ) ^ { 2 } \left(180 x^{4}-360(a+b) x^{3}+300(a+b)^{2} x^{2}-120(a+b)^{3} x\right.\right. \\
& \quad-90(a+b)^{3}+480 x^{3}+(240-720(a+b)) x^{2}+\left(420(a+b)^{2}-240(a+b)-180\right) x \\
& +\left(100(a+b)^{2}+(90+120 a b)(a+b)-160 a b-240 a b x-119\right) \\
& \left.\quad+19(a+b)^{4}-4 a b\left(8(a+b)^{2}+30\left(x^{2}-x(a+b)\right)-a b\right)\right)^{\frac{1}{2}} \\
& \quad \times\left[\frac{1}{b-a} \int_{a}^{b}\left(f^{\Delta \Delta}(t)\right)^{2} \Delta t-\left(\frac{f^{\Delta}(b)-f^{\Delta}(a)}{b-a}\right)^{2}\right]^{\frac{1}{2}} .
\end{aligned}
$$

Čebyšev type inequalities on time scales also hold.

Theorem 3.4. Let $\mathrm{a}, \mathrm{b}, \mathrm{t}, \mathrm{x} \in \mathbb{T}, \mathrm{a}<\mathrm{b}$. Suppose that $\mathrm{f}, \mathrm{g} \in \mathrm{C}_{\mathrm{rd}}^{2}(\mathbb{T}, \mathbb{R})$ are such that

$$
\left\|f^{\Delta \Delta}\right\|_{\infty}:=\sup _{x \in(a, b)}\left|f^{\Delta \Delta}(x)\right|<\infty,\left\|g^{\Delta \Delta}\right\|_{\infty}:=\sup _{x \in(a, b)}\left|g^{\Delta \Delta}(x)\right|<\infty .
$$


Then, the succeeding inequalities hold:

$$
\begin{aligned}
& \mid \frac{1}{b-a} \int_{a}^{b} f(x) g(x) \Delta x-\left(\frac{1}{b-a} \int_{a}^{b} f(x) \Delta x\right)\left(\frac{1}{b-a} \int_{a}^{b} g(x) \Delta x\right) \\
& +\frac{1}{(b-a)^{2}}\left[\left(\int_{a}^{b}\left(x-b+\frac{g_{2}(b, a)}{b-a}\right) g^{\Delta}(x) \Delta x\right)\left(\int_{a}^{b} f(x) \Delta x\right)\right. \\
& \left.+\left(\int_{a}^{b}\left(x-b+\frac{g_{2}(b, a)}{b-a}\right) f^{\Delta}(x) \Delta x\right)\left(\int_{a}^{b} g(x) \Delta x\right)\right] \\
& +\frac{1}{b-a} \int_{a}^{b}\left(x-b+\frac{g_{2}(b, a)}{b-a}\right)^{2} f^{\Delta}(x) g^{\Delta}(x) \Delta x \\
& -\frac{1}{b-a}\left[\int_{a}^{b}\left(x-b+\frac{g_{2}(b, a)}{b-a}\right)\left(f^{\Delta}(x) g(x)+g^{\Delta}(x) f(x)\right) \Delta x\right] \\
& \leqslant \frac{\left\|f^{\Delta \Delta}\right\|_{\infty}\left\|g^{\Delta \Delta}\right\|_{\infty} \int_{a}^{b}\left(h_{2}(b, x)+(x-b) \frac{g_{2}(b, a)}{b-a}+\frac{g_{3}(b, a)}{b-a}\right)^{2} \Delta x,}{b-a} \int_{a}
\end{aligned}
$$

and

$$
\begin{aligned}
& \mid \frac{1}{b-a} \int_{a}^{b} f(x) g(x) \Delta x-\left(\frac{1}{b-a} \int_{a}^{b} f(x) \Delta x\right)\left(\frac{1}{b-a} \int_{a}^{b} g(x) \Delta x\right) \\
& +\frac{1}{b-a}\left[\left(\frac{f(b)-f(a)}{b-a}\right)\left(\frac{g(b)-g(a)}{b-a}\right) \int_{a}^{b}\left(x-b+\frac{g_{2}(b, a)}{b-a}\right)^{2} \Delta x\right] \\
& -\frac{1}{b-a}\left[\frac{g(b)-g(a)}{b-a} \int_{a}^{b}\left(x-b+\frac{g_{2}(b, a)}{b-a}\right) f(x) \Delta x\right. \\
& \left.+\frac{f(b)-f(a)}{b-a} \int_{a}^{b}\left(x-b+\frac{g_{2}(b, a)}{b-a}\right) g(x) \Delta x\right] \mid \\
& \leqslant \frac{\left\|f^{\Delta \Delta}\right\|_{\infty}\left\|g^{\Delta \Delta}\right\|_{\infty} \int_{a}^{b}\left(\int_{a}^{b} \int_{a}^{b}|p(x, \tau)||p(\tau, t)| \Delta t \Delta \tau\right)^{2} \Delta x,}{(b-a)^{5}}
\end{aligned}
$$

where

$$
p(x, t)= \begin{cases}\sigma(t)-a, & t \in[a, x) \\ \sigma(t)-b, & t \in[x, b] .\end{cases}
$$

Proof. We have, from [2, pp. 7], the following identity

$$
\frac{1}{b-a} \int_{a}^{b} f(t) \Delta t+\left(x-b+\frac{g_{2}(b, a)}{b-a}\right) f^{\Delta}(x)-f(x)=\frac{1}{b-a} \int_{a}^{b} u(x, \sigma(t)) f^{\Delta \Delta}(t) \Delta t .
$$


Similarly, one obtains

$$
\frac{1}{b-a} \int_{a}^{b} g(t) \Delta t+\left(x-b+\frac{g_{2}(b, a)}{b-a}\right) g^{\Delta}(x)-g(x)=\frac{1}{b-a} \int_{a}^{b} u(x, \sigma(t)) g^{\Delta \Delta}(t) \Delta t,
$$

where

$$
u(x, \sigma(t))= \begin{cases}g_{2}(t, a), & t \in[a, x), \\ h_{2}(b, t), & t \in[x, b],\end{cases}
$$

and

$$
\frac{1}{b-a} \int_{a}^{b}|u(x, \sigma(t))| \Delta t=\frac{g_{3}(b, a)}{b-a}+(x-b) \frac{g_{2}(b, a)}{b-a}+h_{2}(b, x) .
$$

Now, multiplying both sides of (3.14) and (3.15), we obtain

$$
\begin{aligned}
& f(x) g(x)+\frac{1}{b-a}\left[\left(x-b+\frac{g_{2}(b, a)}{b-a}\right) g^{\Delta}(x) \int_{a}^{b} f(t) \Delta t+\left(x-b+\frac{g_{2}(b, a)}{b-a}\right) f^{\Delta}(x) \int_{a}^{b} g(t) \Delta t\right] \\
& -\frac{1}{b-a}\left[g(x) \int_{a}^{b} f(t) \Delta t+f(x) \int_{a}^{b} g(t) \Delta t\right]+\left(x-b+\frac{g_{2}(b, a)}{b-a}\right)^{2} f^{\Delta}(x) g^{\Delta}(x) \\
& -\left[\left(x-b+\frac{g_{2}(b, a)}{b-a}\right)\left(f^{\Delta}(x) g(x)+g^{\Delta}(x) f(x)\right)\right]+\left(\frac{1}{b-a} \int_{a}^{b} f(t) \Delta t\right)\left(\frac{1}{b-a} \int_{a}^{b} g(t) \Delta t\right) \\
& =\left(\frac{1}{b-a} \int_{a}^{b} u(x, \sigma(t)) f^{\Delta \Delta}(t) \Delta t\right)\left(\frac{1}{b-a} \int_{a}^{b} u(x, \sigma(t)) g^{\Delta \Delta}(t) \Delta t\right) .
\end{aligned}
$$

Integrating both sides of (3.17) with respect to $x$ over $[a, b]$, taking absolute values, using (3.16), and dividing by $(b-a)$, we get the desired inequality (3.12).

We also have (see [2, pp. 14])

$$
f(x)-\frac{1}{b-a} \int_{a}^{b} f(t) \Delta t-\left(x-b+\frac{g_{2}(b, a)}{b-a}\right) \frac{f(b)-f(a)}{b-a}=\frac{1}{(b-a)^{2}} \int_{a}^{b} \int_{a}^{b} p(x, \tau) p(\tau, t) f^{\Delta \Delta}(t) \Delta t \Delta \tau,
$$

and

$$
g(x)-\frac{1}{b-a} \int_{a}^{b} g(t) \Delta t-\left(x-b+\frac{g_{2}(b, a)}{b-a}\right) \frac{g(b)-g(a)}{b-a}=\frac{1}{(b-a)^{2}} \int_{a}^{b} \int_{a}^{b} p(x, \tau) p(\tau, t) g^{\Delta \Delta}(t) \Delta t \Delta \tau,
$$

where

$$
p(x, t)= \begin{cases}\sigma(t)-a, & t \in[a, x), \\ \sigma(t)-b, \& t \in[x, b] . & \end{cases}
$$

From (3.18) and (3.19) we observe that

$$
\begin{aligned}
& f(x) g(x)-\frac{1}{b-a}\left[f(x) \int_{a}^{b} g(t) \Delta t+g(x) \int_{a}^{b} f(t) \Delta t\right] \\
& -\left[\frac{g(b)-g(a)}{b-a}\left(x-b+\frac{g_{2}(b, a)}{b-a}\right) f(x)+\frac{f(b)-f(a)}{b-a}\left(x-b+\frac{g_{2}(b, a)}{b-a}\right) g(x)\right]
\end{aligned}
$$




$$
\begin{aligned}
& +\left(\frac{f(b)-f(a)}{b-a}\right)\left(\frac{g(b)-g(a)}{b-a}\right)\left(x-b+\frac{g_{2}(b, a)}{b-a}\right)^{2} \\
& +\left(\frac{1}{b-a} \int_{a}^{b} f(t) \Delta t\right)\left(\frac{1}{b-a} \int_{a}^{b} g(t) \Delta t\right) \\
& +\frac{1}{b-a}\left[\frac{f(b)-f(a)}{b-a}\left(x-b+\frac{g_{2}(b, a)}{b-a}\right) \int_{a}^{b} g(t) \Delta t+\frac{g(b)-g(a)}{b-a}\left(x-b+\frac{g_{2}(b, a)}{b-a}\right) \int_{a}^{b} f(t) \Delta t\right] \\
& =\frac{1}{(b-a)^{4}}\left(\int_{a}^{b} \int_{a}^{b} p(x, \tau) p(\tau, t) f^{\Delta \Delta}(t) \Delta t \Delta \tau\right)\left(\int_{a}^{b} \int_{a}^{b} p(x, \tau) p(\tau, t) g^{\Delta \Delta}(t) \Delta t \Delta \tau\right) .
\end{aligned}
$$

Integrating both sides of (3.20) with respect to $x$ over $[a, b]$, taking absolute values, and dividing by $(b-a)$, we get the desired inequality (3.13).

Corollary 3.5. Let $\mathbb{T}=\mathbb{R}$ in the Theorem 3.4. Then (3.12) and (3.13) become

$$
\begin{aligned}
& \mid \frac{1}{b-a} \int_{a}^{b} f(x) g(x) d x-\left(\frac{1}{b-a} \int_{a}^{b} f(x) d x\right)\left(\frac{1}{b-a} \int_{a}^{b} g(x) d x\right) \\
& +\frac{1}{(b-a)^{2}}\left[\left(\int_{a}^{b}\left(x-\frac{a+b}{2}\right) g^{\prime}(x) d x\right)\left(\int_{a}^{b} f(x) d x\right)+\left(\int_{a}^{b}\left(x-\frac{a+b}{2}\right) f^{\prime}(x) d x\right)\left(\int_{a}^{b} g(x) d x\right)\right] \\
& +\frac{1}{b-a} \int_{a}^{b}\left(x-\frac{a+b}{2}\right)^{2} f^{\prime}(x) g^{\prime}(x) d x-\frac{1}{b-a}\left[\int_{a}^{b}\left(x-\frac{a+b}{2}\right)(f g)^{\prime} d x\right] \mid \\
& \leqslant \frac{\left\|f^{\prime \prime}\right\|_{\infty}\left\|g^{\prime \prime}\right\|_{\infty}}{60}(b-a)^{4},
\end{aligned}
$$

and

$$
\begin{aligned}
& \mid \frac{1}{b-a} \int_{a}^{b} f(x) g(x) d x-\left(\frac{1}{b-a} \int_{a}^{b} f(x) d x\right)\left(\frac{1}{b-a} \int_{a}^{b} g(x) d x\right) \\
& \quad-\frac{1}{b-a}\left[\frac{g(b)-g(a)}{b-a} \int_{a}^{b}\left(x-\frac{a+b}{2}\right) f(x) d x+\frac{f(b)-f(a)}{b-a} \int_{a}^{b}\left(x-\frac{a+b}{2}\right) g(x) d x\right] \\
& \quad+\left[\frac{(b-a)^{2}}{12}\left(\frac{f(b)-f(a)}{b-a}\right)\left(\frac{g(b)-g(a)}{b-a}\right)\right] \mid \\
& \leqslant \frac{\left\|f^{\prime \prime}\right\|_{\infty}\left\|g^{\prime \prime}\right\|_{\infty}}{(b-a)^{5}} \int_{a}^{b}\left(\int_{a}^{b} \int_{a}^{b}|p(x, \tau)||p(\tau, t)| d t d \tau\right)^{2} d x,
\end{aligned}
$$

where $\left\|f^{\prime \prime}\right\|_{\infty}:=\sup _{x \in(a, b)}\left|f^{\prime \prime}(x)\right|<\infty,\left\|g^{\prime \prime}\right\|_{\infty}:=\sup _{x \in(a, b)}\left|g^{\prime \prime}(x)\right|<\infty$ and

$$
p(x, t)= \begin{cases}t-a, & t \in[a, x) \\ t-b, & t \in[x, b]\end{cases}
$$


Corollary 3.6. Let $\mathbb{T}=\mathbb{Z}$ in the Theorem 3.4. Then (3.12) and (3.13) become

$$
\begin{aligned}
& \mid \frac{1}{b-a} \sum_{x=a}^{b-1} f(x) g(x)-\left(\frac{1}{b-a} \sum_{x=a}^{b-1} f(x)\right)\left(\frac{1}{b-a} \sum_{x=a}^{b-1} g(x)\right) \\
& \quad+\frac{1}{(b-a)^{2}}\left[\left(\sum_{x=a}^{b-1}\left(x-\frac{a+b-1}{2}\right) \Delta g(x)\right)\left(\sum_{x=a}^{b-1} f(x)\right)\right. \\
& \left.\quad+\left(\sum_{x=a}^{b-1}\left(x-\frac{a+b-1}{2}\right) \Delta f(x)\right)\left(\sum_{x=a}^{b-1} g(x)\right)\right]+\frac{1}{b-a} \sum_{x=a}^{b-1}\left(x-\frac{a+b-1}{2}\right)^{2} \Delta f(x) \Delta g(x) \\
& \quad-\frac{1}{b-a}\left[\sum_{x=a}^{b-1}\left(x-\frac{a+b-1}{2}\right)(g(x) \Delta f(x)+f(x) \Delta g(x))\right] \mid \\
& \leqslant
\end{aligned}
$$

and

$$
\begin{aligned}
& \mid \frac{1}{b-a} \sum_{x=a}^{b-1} f(x) g(x)-\left(\frac{1}{b-a} \sum_{x=a}^{b-1} f(x)\right)\left(\frac{1}{b-a} \sum_{x=a}^{b-1} g(x)\right) \\
& +\frac{1}{b-a}\left[\left(\frac{f(b)-f(a)}{b-a}\right)\left(\frac{g(b)-g(a)}{b-a}\right)\left(\frac{(b-a)^{3}-(b-a)}{12}\right)\right] \\
& -\frac{1}{b-a}\left[\frac{g(b)-g(a)}{b-a} \sum_{x=a}^{b-1}\left(x-\frac{a+b-1}{2}\right) f(x)+\frac{f(b)-f(a)}{b-a} \sum_{x=a}^{b-1}\left(x-\frac{a+b-1}{2}\right) g(x)\right] \mid \\
& \leqslant \frac{\left\|\Delta^{2} f\right\|_{\infty}\left\|\Delta^{2} g\right\|_{\infty}}{(b-a)^{5}} \sum_{x=a}^{b-1}\left(\sum_{\tau=a}^{b-1} \sum_{t=a}^{b-1}|p(x, \tau)||p(\tau, t)|\right)^{2},
\end{aligned}
$$

where $\left\|\Delta^{2} f\right\|_{\infty}:=\sup _{a<x<b-1}\left|\Delta^{2} f(x)\right|<\infty,\left\|\Delta^{2} g\right\|_{\infty}:=\sup _{a<x<b-1}\left|\Delta^{2} g(x)\right|<\infty$, and

$$
p(x, t)= \begin{cases}t+1-a, & a \leqslant t<x-1 \\ t+1-b, & x \leqslant t \leqslant b-1\end{cases}
$$

The next theorem contains the Trapezoid type inequality on time scales.

Theorem 3.7. Let $\mathrm{a}, \mathrm{b}, \mathrm{t}, \mathrm{x} \in \mathbb{T}, \mathrm{a}<\mathrm{b}$. Suppose that $\mathrm{f}, \mathrm{g} \in \mathrm{C}_{\mathrm{rd}}^{2}(\mathbb{T}, \mathbb{R})$ are such that

$$
\left\|f^{\Delta \Delta}\right\|_{\infty}:=\sup _{x \in(a, b)}\left|f^{\Delta \Delta}(x)\right|<\infty, \quad\left\|f^{\Delta \Delta}\right\|_{\infty}^{\sigma}:=\sup _{a<x<b}\left|f^{\Delta \Delta}(\sigma(x))\right|<\infty .
$$

Then, we have the succeeding inequality

$$
\begin{aligned}
& \mid \frac{1}{2}\left[f^{2}(b)-f^{2}(a)\right]-\frac{f(b)-f(a)}{2(b-a)} \int_{a}^{b}(f(x)+f(\sigma(x))) \Delta x \\
& \quad-\frac{1}{2} \int_{a}^{b}\left(x-b+\frac{g_{2}(b, a)}{b-a}\right) f^{\Delta}(x)\left(f^{\Delta}(x)+f^{\Delta}(\sigma(x))\right) \Delta x \mid \\
& \leqslant \frac{\left\|f^{\Delta \Delta}\right\|_{\infty}+\left\|f^{\Delta \Delta}\right\|_{\infty}^{\sigma} \int_{a}^{b}\left|f^{\Delta}(x)\right|\left(\frac{g_{3}(b, a)}{b-a}+(x-b) \frac{g_{2}(b, a)}{b-a}+h_{2}(b, x)\right) \Delta x .}{2}
\end{aligned}
$$


Proof. We have (see also [2, pp. 7])

$$
f(x)=\frac{1}{b-a} \int_{a}^{b} f(t) \Delta t+\left(x-b+\frac{g_{2}(b, a)}{b-a}\right) f^{\Delta}(x)-\frac{1}{b-a} \int_{a}^{b} u(x, \sigma(t)) f^{\Delta \Delta}(t) \Delta t,
$$

and

$$
f(\sigma(x))=\frac{1}{b-a} \int_{a}^{b} f(\sigma(t)) \Delta t+\left(x-b+\frac{g_{2}(b, a)}{b-a}\right) f^{\Delta}(\sigma(x))-\frac{1}{b-a} \int_{a}^{b} u(x, \sigma(t)) f^{\Delta \Delta}(\sigma(t)) \Delta t,
$$

where

$$
u(x, \sigma(t))= \begin{cases}g_{2}(t, a), & t \in[a, x) \\ h_{2}(b, t), & t \in[x, b]\end{cases}
$$

Additionally, we have

$$
\frac{1}{b-a} \int_{a}^{b}|u(x, \sigma(t))| \Delta t=\frac{g_{3}(b, a)}{b-a}+(x-b) \frac{g_{2}(b, a)}{b-a}+h_{2}(b, x) .
$$

Adding (3.22) and (3.23), we get

$$
\begin{aligned}
f(x)+f(\sigma(x))= & \frac{1}{b-a} \int_{a}^{b}(f(t)+f(\sigma(t))) \Delta t+\left(x-b+\frac{g_{2}(b, a)}{b-a}\right)\left(f^{\Delta}(x)+f^{\Delta}(\sigma(x))\right) \\
& -\frac{1}{b-a} \int_{a}^{b} u(x, \sigma(t))\left(f^{\Delta \Delta}(t)+f^{\Delta \Delta}(\sigma(t))\right) \Delta t .
\end{aligned}
$$

Multiplying (3.25) by $f^{\Delta}(x)$, using Theorem 2.6, and integrating the resultant identity on $[a, b]$ we get

$$
\begin{aligned}
f^{2}(b)-f^{2}(a)= & \frac{f(b)-f(a)}{b-a} \int_{a}^{b}(f(x)+f(\sigma(x))) \Delta x+\int_{a}^{b}\left(x-b+\frac{g_{2}(b, a)}{b-a}\right) f^{\Delta}(x)\left(f^{\Delta}(x)+f^{\Delta}(\sigma(x))\right) \Delta x \\
& -\frac{1}{b-a} \int_{a}^{b} f^{\Delta}(x)\left(\int_{a}^{b} u(x, \sigma(t))\left(f^{\Delta \Delta}(t)+f^{\Delta \Delta}(\sigma(t))\right) \Delta t\right) \Delta x .
\end{aligned}
$$

Taking absolute values of the above equation and using (3.24), we therefore arrive at the intended inequality.

Corollary 3.8. If we let $\mathbb{T}=\mathbb{R}$ in the Theorem 3.7, then (3.21) becomes

$$
\begin{aligned}
& \left|\frac{1}{2}\left[f^{2}(b)-f^{2}(a)\right]-\frac{f(b)-f(a)}{b-a} \int_{a}^{b} f(x) d x-\int_{a}^{b}\left(x-\frac{a+b}{2}\right)\left(f^{\prime}(x)\right)^{2} d x\right| \\
& \quad \leqslant \frac{\left\|f^{\prime \prime}\right\|_{\infty}}{2} \int_{a}^{b}\left|f^{\prime}(x)\right|\left(\left(x-\frac{a+b}{2}\right)^{2}+\frac{(b-a)^{2}}{12}\right) d x,
\end{aligned}
$$

where $\left\|f^{\prime \prime}\right\|_{\infty}:=\sup _{a<x<b}\left|f^{\prime \prime}(x)\right|<\infty$. This inequality is the Trapezoid type inequality given by Pachpatte [18, Theorem $\left.1\left(\mathrm{a}_{2}\right)\right]$. 
Corollary 3.9. If we let $\mathbb{T}=\mathbb{Z}$ in the Theorem 3.7 , then (3.21) becomes

$$
\begin{aligned}
\mid \frac{1}{2}\left[f^{2}(b)-f^{2}(a)\right]-\frac{f(b)-f(a)}{2(b-a)} \sum_{x=a}^{b-1}(f(x)+f(x+1)) \\
\quad-\frac{1}{2} \sum_{x=a}^{b-1}\left(x-\frac{a+b-1}{2}\right)(\Delta f(x))(\Delta f(x)+\Delta f(x+1)) \mid \\
\leqslant \frac{\left\|\Delta^{2} f\right\|_{\infty}+\left\|\Delta^{2} f\right\|_{\infty}^{\sigma} \sum_{x=a}^{b-1}|\Delta f(x)|\left(\left(x+1-\frac{a+b}{2}\right)^{2}+\frac{(b-a+2)(b-a-2)}{12}\right),}{2}
\end{aligned}
$$

where $\left\|\Delta^{2} f\right\|_{\infty}:=\sup _{\mathbf{a}<x<b-1}\left|\Delta^{2} f(x)\right|<\infty$ and $\left\|\Delta^{2} f\right\|_{\infty}^{\sigma}:=\sup _{a<x<b-1}\left|\Delta^{2} f(x+1)\right|<\infty$.

\section{Conclusion}

In this article, inequalities of the Ostrowski, Ostrowski-Grüss, and Trapeziod kinds on time scales are hereby presented. By employing our results to the continuous and discrete calculus, more interesting inequalities are obtained as special cases. Theorem 3.7 extends the Trapezoid type inequality given by Pachpatte [18] to time scales. More results can be obtained by applying our results to the time scale $\mathbb{T}=q^{\mathbb{N}}$, where $\mathrm{q}>1$.

\section{Acknowledgment}

We appreciate the suggestions of the anonymous referee.

\section{References}

[1] R. Agarwal, M. Bohner, A. Peterson, Inequalities on time scales: a survey, Math. Inequal. Appl., 4 (2001), 535-557. 2

[2] E. Akin-Bohner, M. Bohner, T. Matthews, Time scales Ostrowski and Grüss type inequalities involving three functions, Nonlinear Dyn. Syst. Theory, 12 (2012), 119-135. 3, 3, 3, 3, 3

[3] M. Bohner, T. Matthews, Ostrowski inequalities on time scales, JIPAM. J. Inequal. Pure Appl. Math., 9 (2008), 8 pages. 1

[4] M. Bohner, E. R. Nwaeze, A. Tuna, Trapezoid-Type Inequalities on Time Scales, Submitted. 1, 2

[5] M. Bohner, A. Peterson, Advances in dynamic equations on time scales, Birkhäuser Boston, Boston, (2003). 2

[6] M. Bohner, A. Peterson, Dynamic Equations on Time Scales: An Introduction with Applications, Birkhäuser Boston, Boston, (2001). 2

[7] P. L. Čebyšev, Sue les expressions approxmatives des intégrales définies par les autres prises entre les mêmes limites, Proc. Math. Soc. Charkov, 2 (1882), 93-98. 1

[8] A. A. El-Deeb, H. A. Elsennary, E. R. Nwaeze, Generalized Weighted Ostrowski, Trapezoid and Grüss Type Inequalities on Time Scales, Fasc. Math., 60 (2018), 123-144. 1

[9] S. Hilger, Ein Maßkettenkalkül mit Anwendung auf Zentrumsmannigfaltigkeiten, Ph.D. Thesis, Universität Würzburg, Würzburg, Germany, (1988). 1, 2

[10] V. Lakshmikantham, S. Sivasundaram, B. Kaymakcalan, Dynamic systems on measure chains, Kluwer Academic Publishers Group, Dordrecht, (1996). 2

[11] W. J. Liu, Q.-A. Ngô, W. B. Chen, A new generalization of Ostrowski type inequality on time scales, An. Şt. Univ. Ovidius Constanţa, 17 (2009), 101-114. 1

[12] W. J. Liu, A. Tuna, Diamond weighted Ostrowski type and Grüss type inequalities on time scales, Appl. Math. Comput., 270 (2015), 251-260.

[13] W. J. Liu, A. Tuna, Weighted Ostrowski, Trapezoid and Grüss type inequalities on time scales, J. Math. Inequal., 6 (2012), 381-399.

[14] W. J. Liu, A. Tuna, Y. Jiang, New weighted Ostrowski and Ostrowski-Grüss type inequalities on time scales, An. Ştiinţ. Univ. Al. I. Cuza Iaşi. Mat. (N.S.), 60 (2014), 57-76.

[15] W. J. Liu, A. Tuna, Y. Jiang, On weighted Ostrowski type, Trapezoid type, Grüss type and Ostrowski-Grüss like inequalities on time scales, Appl. Anal., 93 (2014), 551-571.

[16] E. R. Nwaeze, Generalized weighted trapezoid and Grüss type inequalities on time scales, Aust. J. Math. Anal. Appl., 14 (2017), 13 pages. 1 
[17] A. Ostrowski, Uber die Absolutabweichung einer differentiierbaren Funktion von ihrem Integralmittelwert, Comment. Math. Helv., 10 (1937), 226-227. 1

[18] B. G. Pachpatte, On trapezoid and Grüss-like integral inequalities, Tamkang J. Math., 34 (2003), 365-369. 3.8, 4

[19] A. Tuna, Y. Jiang, W. J. Liu, Weighted Ostrowski, Ostrowski-Grüss and Ostrowski-Čebyšev Type Inequalities on Time Scales, Publ. Math. Debrecen, 81 (2012), 81-102. 1

[20] A. Tuna, W. Liu, New weighted Čebyšev-Ostrowski type integral inequalities on time scales, J. Math. Inequal., 10 (2016), 327-356.

[21] G. P. Xu, Z. B. Fang, A New Ostrowski type inequality on time scales, J. Math. Inequal., 10 (2016), 751-760. 1 